\title{
Study on Filtration Device Using Nanoparticles and Its Characteristics for Molecular Separation
}

\author{
Jae Sung Yoon ${ }^{1,2}$, Nguyen Thi Phuong ${ }^{1,2}$, Jeong Hwan Kim ${ }^{1,2}$, Kwanoh Kim ${ }^{1}$, Doo-Sun Choi ${ }^{1,2}$, \\ Seungmin Hyun ${ }^{1}$, Yeong-Eun Yoo ${ }^{1,2}$ \\ ${ }^{1}$ Department of Nano Manufacturing Technology, Korea Institute of Machinery \& Materials (KIMM) \\ Daejeon 34103, Korea \\ jaesyoon@kimm.re.kr \\ ${ }^{2}$ Department of Nano Mechatronics, Korea University of Science and Technology (UST) \\ Daejeon 34113, Korea
}

\section{Extended Abstract}

Since the importance of water treatment and reuse is becoming greater, a lot of filtration devices and membranes have been developed so far. And technical demands for finer filtration, or separation, have been increased recently, so many researchers have focused on achieving smaller filtration sizes with filters, membranes and related devices, accordingly. Although modern fabrication techniques have shown various opportunities for that purpose, it is still challenging to fabricate pores or channels for filtration or separation in nanoscale.

Meanwhile, it is well known that sand bed has voids inside and the voids are much smaller than the sand particles. Therefore, the sand bed has been utilized as filtration media for a long time and it is still used as basic principle for pretreatment of water in modern industries [1,2]. This study provides a new principle for fabrication of nano voids using nanoparticles, in the similar way of sand bed. Polystyrene nanoparticles with various sizes of 150, 60 and $24 \mathrm{~nm}$ have been embedded in the pores of the anodic aluminum oxide (AAO) template, so that voids between the nanoparticles could be made in nano scale. These voids can be considered as effective pores for filtration or separation [3]. By this method, a lot of nano voids could be fabricated on a large area (diameter of template : $25 \mathrm{~mm}$ ) with a simple process, which may be an important advantage for mass production in the future.

Since overall size of the voids is expected to be much smaller than that of the nanoparticles, this principle is expected to have effective separation size of a few nanometers. And as long as the size of the nanoparticles are uniform, size of the voids could be quite uniform also. Furthermore, we treated the surfaces of the nanoparticles with arginine/phenylalanine peptide $((\mathrm{RF}) 4)$, so that the particles could be electrically charged [4,5]. By this way, the filtration device in this study could have separation characteristics of electric charge as well as size.

In order to evaluate embedment of the nanoparticles, water flow was applied across the device and pressure drops were measured. The pressure drops became higher as smaller nanoparticles were embedded, which means that smaller voids were made inside. And separation experiments were also performed with electrically charged dye molecule, which is methylene blue. Experimental results have also shown that higher separation rate was achieved as smaller nanoparticles were used. And the effect of the surface charge on separation performance was also measured. Positively charged nanoparticles have produced higher separation, or rejection, for positively charged methylene blue molecules. On the other hand, negatively charged nanoparticles have produced lower separation. These results show that filtration device in this study has both characteristics of size separation and charge separation. Consequently, this study is expected to provide a new principle for filtration or separation technology in nanoscale, which is also feasible for molecules, as well as the fabrication technology for nano voids or nanopores.

\section{References}

[1] C. Vohla, M. Kõiv, H. J. Bavor, F. Chazarenc, Ü. Mander, "Filter materials for phosphorus removal from wastewater in treatment wetlands-A review," Ecological Engineering, vol. 39, pp. 70-89, 2011. 
[2] A. M. Nassar, K. Hajjaj, "Purification of stormwater using sand filter," J. Water Res. Protect., vol. 5, pp. 1007-1012, 2013.

[3] J. S. Yoon, et al., "Method for manufacturing nanoholes and filter manufactured by the same," Korea Patent 1605632, 2015, European Patent 15153186.0, 2015, Singapore Patent 10201500789X, 2015.

[4] M. S. Liberato, S. Kogikoski, Jr., E. R. Silva, M. D. C. Neto, L. P. B. Scott, R. H. Silva, V. X. Oliveira, Jr., R. A. Ando, W. A. Alves, "Self-Assembly of Arg-Phe Nanostructures via the Solid-Vapor Phase Method," J. Phys. Chem. vol. 117, pp. 733-740, 2013.

[5] C. C. Decandio, E. R. Silva, I. W. Hamley, V. Castelletto, M. S. Liberato, V. X. Oliveira, Jr., C. L. P. Oliveira, W. A. Alves, "Self-Assembly of a Designed Alternating Arginine/Phenylalanine Oligopeptide," Langmuir, vol. 31, pp. 4513-4523, 2015. 